Results She showed complete recovery after rituximab.

Conclusions Rituximab can be considered as an effective treatment modality in APLA related chorea where conventional measures have failed.

\section{DYSBIOSIS AND GUT BARRIER DYSFUNCTION IN ANTIPHOSPHOLIPID SYNDROME AS REVEALED BY IGA- SEQ PROFILING}

$1,{ }^{2}$ WJ Kim* ${ }^{2}$ W Ruff, ${ }^{3} \mathrm{C}$ Aguiar, ${ }^{2} \mathrm{~A}$ Yu, ${ }^{2} \mathrm{SM}$ Vieira, ${ }^{2} \mathrm{JA}$ Sterpka, ${ }^{4} \mathrm{~A}$ Goodman, ${ }^{3} \mathrm{D}$ Erkan, 2,5 MA Kriegel. 'Cheju Halla General Hospital, Laboratory Medicine, Jeju-si, Republic of Korea; ${ }^{2}$ Yale School of Medicine, Immunobiology, New Haven, USA; ${ }^{3}$ Hospital for Special Surgery, Medicine, NewYork, USA; ${ }^{4}$ Yale School of Medicine, Microbial Pathogenesis, New Haven, USA; ${ }^{5}$ Yale School of Medicine, Medicine, New Haven, USA

\subsection{6/lupus-2017-000215.64}

Background and aims The antiphospholipid syndrome (APS) is an autoimmune thrombophilic non-gut disorder with high mortality. Various pathogens have been associated with transient antiphospholipid antibody production. We hypothesised that members of the gut microbiota in APS patients could represent a chronic trigger and exhibit heightened adaptive immune responses to the microbiota. The purpose of this study was to explore gut barrier function and faecal IgAcoated microbial composition in APS patients.

Methods Stool from 15 APS patients, 5 non-autoimmune thrombotic states, and 12 normal donors (total of 17 controls) was collected. Faecal homogenates were analysed for the gut permeability marker calprotectin and, in parallel, stained stained with PE-conjugated anti-human IgA prior to cell sorting. Faecal DNA was isolated and PCR-amplified targeting the V4 region of the $16 \mathrm{~S}$ rRNA gene. Samples were sequenced on the Illumina MiSeq platform.

Results Faecal calprotectin and IgA-coated faecal bacterial levels were significantly higher in APS patients compared to controls $(\mathrm{p}<0.003 ; \mathrm{p}<0.05)$. LEfSe analysis of IgA+ fractions showed that the strongest IgA-coated genus is Blautia in APS.

Conclusions These data suggest gut barrier dysfunction and aberrant IgA coating of commensals in APS. Markedly enhanced bacterial IgA coating in several APS patients supports a stronger adaptive immune response to the microbiota. Increased IgA coating of Blautia might reflect altered gut homeostasis as a Blautia species was shown to be part of proinflammatory $\operatorname{IgA}+$ consortium in a recent study in IBD. To our knowledge, this study represents the first 16S rRNA profiling of IgA-coated gut commensals in patients with nongut autoimmunity.

\section{ANTIPHOSPHOLIPID SYNDROME LONG TERM FOLLOW UP: REAL LIFE EXPERIENCE OF A SINGLE CENTRE}

${ }^{1} \mathrm{RM}$ Serrano Morales*${ }^{*}{ }^{2} \mathrm{G}$ Pons-Estel, ${ }^{3} \mathrm{R}$ Quintana, ${ }^{2} \mathrm{G}$ Espinosa Garriga, ${ }^{4} \mathrm{R}$ Cervera Segura. 'Barcelona, Spain; ${ }^{2}$ Hospital Clínic, Autoimmune Diseases Unit, Barcelona, Spain; ${ }^{3}$ Hospital Provincial de Rosario, Reumatología, Rosario, Argentina; ${ }^{4}$ Hospital Clínic Barcelona, Autoimmune Diseases Unit, Barcelona, Spain

\subsection{6/lupus-2017-000215.65}

Background and aims The antiphospholipid syndrome (APS) has wide clinical and outcome spectrum overtime. The aim of this study was to assess the real life prevalence of morbi-mortality APS during 10 year-follow-up of a single referral centre.
Methods The cohort included 160 consecutive APS patients followed by the Autoimmune Diseases Unit of Hospital Clínic Barcelona from 2003 to 2013. Epidemiological, clinical, immunological and treatment features were analysed prospectively.

Results The cohort consisted of 126 (79\%) women and 34 (21\%) mens. Mean (SD) age at diagnosis was 39 (14) years. The diagnostic causes were thrombosis $(56.3 \%)$, obstetric morbidity (26.9\%) and both (16.9\%). 65\% were primary APS, $22.5 \%$ associated with systemic lupus erythematosus (SLE), $8.8 \%$ associated with lupus-like syndrome and $3.7 \%$ associated with other diseases. Fifty-five patients were lost to follow-up (3.4\% every year). In evolution, 10 primary APS patients were reclassified as SLE-associated APS and 2 patients developed catastrophic APS. Table 1 shows the frequencies of the main APS clinical manifestations at baseline and during the 10-year-follow-up. At diagnosis, 95\% received antithrombotic treatment: low dose antiaggregants (39.5\%), oral anticoagulants $(67.1 \%)$, heparin $(2.6 \%)$. During the study, $72.7 \%$ of recurrences were without antithrombotic treatment and $27.3 \%$ were despite it. Eleven major bleeding episodes occurred and 2 were fatal. The global mortality rate was $6.9 \%$ and $50 \%$ in catastrophic APS. Table 2 shows the main causes of death and Figure 1 is a Kaplan-Meier survival curve.

Conclusions This study shows long-term morbidity and mortality of a large APS patient cohort and exposed the real-life experience of a referral unit.

\section{ANTIPHOSPHOLIPID SYNDROME - CINICAL AND IMMUNOLOGICAL CORRELATIONS AND ATHEROSCLEROSIS}

N Stoilov*, V Boyadzhieva, R Rashkov. University hospital "St. Ivan Rilski", Clinic of Rheumatology, Sofia, Bulgaria

\subsection{6/lupus-2017-000215.66}

Background and aims In recent years, it is found that role in atherogenesis plays inflammatory component of immune response. In recent years found unarguably data for accelerated development of atherosclerotic plaques in patients with APS.

Our aim is to investigate the frequency of cardiovascular events and atherosclerosis in patients with primary and secondary antiphospholipid syndrome compared to healthy individuals and patients with systemic lupus erythematosus without antiphospholipid antibodies.

Methods We studied 99 patients with APS, 13 SLE and 32 healthy controls. They were tested for aPL antibodies (aCL, anti-b2gp1, anti-prothrombin), ANA-screen, ANA - profile and standart laboratory.

We examine Intima-Media thickness of carotit arteries and Calcium score of: a. coronaria sinister, a. anterior descendens sinister, a. circumflexa sinister, a. coronaria dexter, Aorta, Valvaorte to validate the atheroslerosis.

Results We found strong, statistically significant correlation between aCL antibodies and the presence of plaques in the left common carotid artery $(\mathrm{p}=0.041)$ and absent a correlation between aPL titers and presence of carotid plaques. In the group with APS, 33,3\% (14) establishes a positive calcium score of coronary artheries, $11.9 \%$ (5) positive for aorta, Aortic valve Absent deposits, In the control group positive calcium score is one person $(5.88 \%)$. 